\title{
PADRÕES DE COMÉRCIO INTERESTADUAL NO BRASIL, 1985 E 1997*
}

\author{
Fernando Salgueiro Perobelli ${ }^{* *}$
}

\section{Eduardo Amaral Haddad ${ }^{* * *}$}

RESUMO O principal objetivo deste trabalho é verificar o padrão de comércio entre as 27 unidades da Federação para os anos de 1985 e 1997. A fim de implementar tal análise foram utilizados dados dos fluxos de comércio inter-regional (CONFAZ e IBGE). A análise espacial permite detectar a presença de autocorrelação espacial global e local na distribuição de comércio e em seus componentes. Os resultados da estatística LISA nos permitem observar a existência de heterogeneidade espacial no comércio inter-regional durante o período de análise. A existência de clusters de alto comércio e baixo comércio inter-regional durante o período de análise pode indicar a persistência de disparidades regionais entre as unidades da Federação no curto prazo.

Palavras-chave: análise exploratória espacial; autocorrelação espacial; heterogeneidade espacial e economia brasileira

Código JeL: O18, O54, R11, R12

THE BRAZILIAN INTER-REGIONAL TRADE PATTERN: 1985 E 1997

ABSTRACT The aim of this paper is explore the spatial distribution of the interregional trade among the 27 Brazilian states for the years 1985 and 1997. To implement this methodology we use, as a dataset, the interregional exports and imports

* Artigo recebido em 16 de setembro de 2004 e aprovado em 15 de dezembro de 2005. Os autores agradecem os comentários de Julie Le Gallo, Mônica Haddad e Rangel Galinari em versões anteriores deste texto e aos pareceristas anônimos da Revista de Economia Contemporânea, eximindo-os de qualquer erro. Do mesmo modo mostram-se reconhecidos ao financiamento da CAPES e do CNPq, concedido ao primeiro e segundo autores, respectivamente.

** Coordenador e professor de mestrado em Economia Aplicada da FEA/UFJF e pesquisador do NEREUS/USP, e-mail: fernando.perobelli@ufjf.edu.br

*** Professor do IPE/USP e pesquisador do NEREUS/USP, bolsista do CNPq, e-mail: ehaddad@usp.br 
flows (CONFAZ and IBGE). Based on a set of tools of spatial analysis it is possible to detect the presence of global and local spatial autocorrelation in the distribution of trade and its components. The results of LISA statistics enable us to observe the presence of spatial heterogeneity in the interregional trade during the period of analysis. This paper concludes that the detection of spatial clusters of high and low interregional trade throughout the period is an indication of the persistence of spatial disparities among the Brazilian states.

Key words: exploratory spatial analysis; spatial autocorrelation; spatial heterogeneity and Brazilian economy 


\section{INTRODUÇÃO}

O objetivo deste trabalho é estudar, com maior grau de detalhamento, os fluxos de comércio inter-regional nos anos de 1985 e 1997 e, portanto, ter uma idéia da evolução do grau de integração do mercado nacional. Em outras palavras, busca-se verificar qual é a dinâmica espacial do comércio interregional brasileiro, ou seja, trabalhar os aspectos espaciais da base de dados, explicitando se há regimes espaciais de comércio, comportamento atípico das unidades da Federação, formação de clusters de comércio e classificação de tais clusters (e.g. regiões de alto comércio vizinhas de regiões de alto comércio, regiões de baixo comércio vizinhas de regiões de baixo comércio, regiões de alto comércio vizinhas de regiões de baixo comércio etc.).

Portanto, não é objetivo deste trabalho fazer uma análise comparativa com os demais da literatura, que tratam da questão do comércio inter-regional. Entre os trabalhos sobre o tema destacam-se: (a) Galvão (1999 e 1993), que estuda o comércio interestadual no período 1948-1969, (b) Diniz e Lemos (1989), que analisam os fluxos inter-regionais para o período 19701980, (c) Pacheco (1998), que, a partir dos dados das balanças comerciais interestaduais, faz uma análise para o período 1975-1985, e (d) Domingues et al. (2002), os quais examinam as mudanças nos fluxos de comércio interregional brasileiro, para o período 1985-1997.

Cabe aqui dar maior ênfase aos resultados encontrados por Pacheco (1998) e Domingues et al. (2002), pois, apesar de usarem metodologias diferentes daquela apresentada no presente artigo, utilizam a mesma base de dados para o ano de 1985, no caso de Pacheco (1998), e no caso de Domingues et al. (2002), tanto para o ano de 1985 como para 1997.

Pacheco (1998) analisa o comércio inter-regional a partir de três indicadores: (a) coeficientes de comércio inter-regional em percentagem do PIB, (b) participação do comércio inter-regional no comércio interestadual e (c) balança comercial em termos do PIB de cada unidade da Federação. A análise de Pacheco (1998) para os dados do ano de 1985 mostra que (1) as exportações de São Paulo para as demais unidades da Federação em 1985 eram 30,7\% do PIB e as exportações do resto do Brasil para São Paulo eram $13,2 \%$ do PIB. Isso reflete a importância do mercado paulista enquanto destino da produção dos bens produzidos nas demais unidades da Federação; e (2) em geral, a participação do comércio inter-regional, em termos do 
conjunto das vendas interestaduais das unidades da Federação de uma determinada macrorregião, tem aumentado, isto é, a importância do comércio inter-regional diante das operações intra-regionais torna-se maior. No caso das exportações, a participação inter-regional para o Norte é de $88 \%$ do comércio interestadual e 59,6\% para o Nordeste.

Domingues et al. (2002), como afirmamos anteriormente, usam a mesma base de dados do presente artigo, embora utilizem metodologia diferente (e.g. modelo gravitacional e análise de cluster). Os autores captam a questão espacial no modelo gravitacional utilizando apenas uma dummy de região. Domingues et al. (2002) mostram (a) a importância dos estados maiores (em termos de PIB) no comércio inter-regional; (b) o papel da distância na determinação dos fluxos de comércio. Segundo os autores, utilizando um modelo gravitacional, ainda é possível verificar que os estados vizinhos têm um comércio maior; (c) que há maior similaridade no padrão de comércio entre os maiores estados; e (d) que São Paulo exibe um comportamento distinto dos demais estados.

Logo, este artigo tem caráter complementar à análise realizada pelos autores citados, como também visa preencher a lacuna existente na literatura brasileira para o período recente e, assim, contribuir para o melhor entendimento das questões comerciais das unidades da Federação.

Com base nas contas estaduais construídas por Haddad et al. (2002) para o ano de 1996, pode-se perceber que: (a) para os estados do Sudeste e do Sul, a participação das exportações totais na formação do PRB (Produto Regional Bruto) é maior do que a participação das importações; (b) para todas as unidades da Federação o comércio inter-regional (exportações e importações) é mais importante do que o comércio internacional, em termos de formação do PRB; e (c) todas as unidades da Federação localizadas nas regiões Sudeste e Sul, com exceção de Minas Gerais, apresentam resultados superavitários na balança comercial total, ao passo que as unidades da Federação localizadas nas regiões Norte, Nordeste e Centro-Oeste apresentam déficit na balança comercial.

Assim sendo, a análise da questão das disparidades espaciais do comércio entre as unidades da Federação é realizada, neste trabalho, a partir da análise exploratória de dados espaciais (AEDE), ou seja, a partir da análise da autocorrelação espacial, da heterogeneidade espacial, dos clusters de co- 
mércio (e.g. alta intensidade de importações e exportações) para o comércio inter-regional brasileiro, para os anos de 1985 e 1997. A partir da AEDE objetiva-se incorporar os problemas locacionais na discussão sobre comércio. Tal discussão é muito importante para a análise da performance econômica das regiões.

O problema locacional possibilita trabalhar com dois efeitos espaciais diferentes, a saber, dependência espacial e heterogeneidade espacial. A dependência espacial está diretamente relacionada à primeira lei da geografia. ${ }^{1}$ Tomando por base essa lei, é possível afirmar que as observações estão espacialmente aglomeradas, conseqüentemente, há clusters. Assim, os dados geográficos não serão independentes. É importante salientar que, sob a perspectiva geográfica, a dependência espacial é uma regra. A dependência dos dados espaciais pode ser classificada como autocorrelação espacial. Portanto, localizações similares (e.g. observações que apresentam um certo grau de proximidade espacial) apresentam valores similares (correlação). Logo, haverá autocorrelação positiva quando valores altos ou baixos de uma variável aleatória específica formarem clusters espaciais, e autocorrelação espacial negativa quando os vizinhos de uma determinada área geográfica apresentarem valores diferentes.

O conceito de heterogeneidade espacial está relacionado com a idéia de instabilidade do comportamento econômico no espaço. Em outras palavras, liga-se à diferenciação espacial ou regional, ou seja, à concepção de que existem características intrínsecas a cada região. Em conseqüência, é possível haver diversos padrões espaciais no desenvolvimento econômico. Então, os resultados podem apresentar regimes espaciais (e.g. um cluster com regiões desenvolvidas, centro, ou um cluster com regiões menos desenvolvidas, periferia) (Le Gallo e Ertur, 2003).

Assim sendo, a análise do padrão de comércio entre as unidades da Federação para os anos de 1985 e 1997 realiza-se pelas medidas de autocorrelação espacial global e autocorrelação espacial local. A matriz de transição de probabilidades é utilizada para verificar a robustez dos resultados da associação espacial local. Para tal, o artigo, além desta parte introdutória, apresenta de forma breve a metodologia utilizada, na seção 1 . Na seção 2 os principais resultados encontrados são discutidos e na seção 3 apresentam-se as considerações finais. 


\section{METODOLOGIA}

A AEDE está baseada nos aspectos espaciais da base de dados, ou seja, trata diretamente de questões como dependência espacial (e.g. associação espacial) e heterogeneidade espacial. Em outros termos, o objetivo deste método é descrever a distribuição espacial, os padrões de associação espacial (clusters espaciais), verificar a existência de diferentes regimes espaciais ou outras formas de instabilidade espacial (não-estacionariedade) e identificar observações atípicas (i.e., outliers). A partir disso é possível extrair medidas de autocorrelação espacial e autocorrelação local (Anselin, 1998).

\subsection{Autocorrelação espacial global}

A autocorrelação espacial pode ser calculada a partir da estatística I de Moran. Essa estatística fornece a indicação formal do grau de associação linear entre os vetores de valores observados no tempo $t\left(z_{t}\right)$ e a média ponderada dos valores da vizinhança, ou seja, os lags espaciais $\left(W z_{t}\right){ }^{2}$ Valores de I maiores (ou menores) do que o valor esperado $E(I)=-1 /(n-1)$ significa que há autocorrelação positiva (ou negativa). Seguindo Cliff e Ord (1981), em termos formais, a estatística $I$ de Moran pode ser expressa da seguinte forma:

$$
I_{t}=\left(\frac{n}{S_{o}}\right)\left(\frac{z_{t}^{\prime} W z_{t}}{z_{t}^{\prime} z_{t}}\right) t=1,(\ldots) n
$$

onde $z_{t}$ é o vetor de $n$ observações para o ano $t$ na forma de desvio em relação à média. $W$ é a matriz de pesos espaciais: os elementos $w_{i i}$ na diagonal são iguais a zero, enquanto os elementos $w_{i j}$ indicam a forma como a região $i$ está espacialmente conectada com a região $j$. $S_{o}$ é um escalar igual à soma de todos os elementos de $W$.

Quando a matriz de pesos espaciais é normalizada na linha, isto é, quando os elementos de cada linha somam 1, a expressão (1) toma a seguinte forma:

$$
I_{t}=\left(\frac{z_{t}{ }^{\prime} W z_{t}}{z_{t}^{\prime} z_{t}}\right) t=1,(\ldots) n
$$

É importante ressaltar que a estatística I de Moran é uma medida global, portanto não é possível observar a estrutura de correlação espacial em nível regional. 


\subsection{Autocorrelação espacial local}

A fim de observar a existência de clusters espaciais locais de valores altos ou baixos e quais as regiões que mais contribuem para a existência de autocorrelação espacial, devem-se implementar as medidas de autocorrelação espacial local, quais sejam: gráfico de dispersão de Moran e LISA (Indicadores Locais de Associação Espacial).

\subsubsection{Gráficos de dispersão de Moran}

Segundo Anselin (1996), o gráfico de dispersão de Moran é uma das formas de interpretar a estatística I de Moran. Em outras palavras, é uma representação do coeficiente de regressão e permite visualizar a correlação linear entre $z$ e $W z$ a partir do gráfico de duas variáveis. No caso específico da estatística $I$ de Moran, tem-se o gráfico de Wz e $z$. Portanto, o coeficiente $I$ de Moran é a inclinação da curva de regressão e essa inclinação indica o grau de ajustamento.

O gráfico de dispersão de Moran é dividido em quatro quadrantes. Esses quadrantes correspondem a quatro padrões de associação local espacial entre as regiões e seus vizinhos.

O primeiro quadrante (localizado na parte superior direita) mostra as regiões que apresentam altos valores para a variável em análise (e.g. valores acima da média) cercadas por regiões que também apresentam valores acima da média para a variável em análise. A classificação atribuída a esse quadrante é alto-alto $(A A)$.

O segundo quadrante (localizado na parte superior esquerda) mostra as regiões com valores baixos cercados por vizinhos que apresentam valores altos. Esse quadrante é geralmente classificado como baixo-alto $(B A)$.

O terceiro quadrante (localizado no canto inferior esquerdo) é constituído pelas regiões com valores baixos para as variáveis em análise cercados por vizinhos que também apresentam baixos valores. Esse quadrante é identificado como baixo-baixo $(B B)$.

O quarto quadrante (localizado no canto inferior direito) é formado pelas regiões com altos valores para as variáveis em análise cercadas por regiões com baixos valores. Tal quadrante classifica-se como alto-baixo $(A B)$.

As regiões que estão localizadas nos quadrantes $A A$ e $B B$ apresentam autocorrelação espacial positiva, ou seja, essas regiões formam clusters de valores 
similares. Por outro lado, os quadrantes $B A$ e $A B$ apresentam autocorrelação espacial negativa, logo, essas regiões formam clusters com valores diferentes.

\subsubsection{Indicadores locais de associação espacial (LISA)}

Segundo Anselin (1995), um "Indicador Local de Associação Espacial (LISA)" é qualquer estatística que satisfaça dois critérios: (a) um indicador LISA deve possuir, para cada observação, uma indicação de clusters espaciais significantes de valores similares em torno da observação (e.g. região) e (b) o somatório dos LISAs, para todas as regiões, é proporcional ao indicador de autocorrelação espacial global.

De acordo com Le Gallo e Ertur (2003), a estatística LISA pode ser especificada da seguinte forma:

$$
I_{i, t}=\frac{\left(x_{i, t}-\mu_{t}\right)}{m_{o}} \sum_{j} w_{i j}(x-\mu) \operatorname{com} m_{o}=\frac{\left(x_{i, t}-\mu\right)^{2}}{n}
$$

onde $x_{i, t}$ é a observação na região $i$ para o ano te $\mu_{t}$ é a média das observações entre as regiões no ano $t$ para a qual o somatório em relação a $j$ é tal que somente os valores vizinhos de $j$ são incluídos.

A estatística pode ser interpretada da seguinte forma: valores positivos de $I_{i, t}$ significam que existem clusters espaciais com valores similares (alto ou baixo); valores negativos significam que existem clusters espaciais com valores diferentes entre as regiões e seus vizinhos.

De acordo com Anselin (1995), a estatística LISA é utilizada para medir a hipótese nula de associação espacial local. É importante salientar que assim como a distribuição para as estatísticas globais, a distribuição genérica para a estatística LISA também é de difícil apuração. Portanto, para solucionar o problema deve-se trabalhar com resultados assintóticos. Assim, a alternativa é a utilização de uma randomização condicional ou uma permutação que permita auferir pseudoníveis de significância. Os níveis de significância das distribuições marginais são aproximados a partir das desigualdades de Bonferroni ou a partir da estrutura proposta por Sidák (1967) apud Anselin (1995). "O significado disso é que a variância total associada com as comparações múltiplas (testes de correlação) é definida como $\alpha$, e existem $m$ comparações. Logo, a significância individual $\alpha_{i}$ poderia ser definida como $\alpha / m$ (Bonferroni) ou $1-(1-\alpha)^{1 / m}$ (Sidák)" (Anselin, 1995: 96). Neste trabalho é utilizada a análise de Bonferroni. ${ }^{3}$ 


\subsection{Determinação da matriz de pesos espaciais}

A matriz de peso é a forma de expressar a estrutura espacial (e.g. continuidade) dos dados, sendo o ponto inicial para qualquer teste estatístico ou modelo. Há na literatura um grande número de matrizes de peso. É possível implementar um AEDE com base em uma matriz de continuidade binária ou a partir de uma estrutura mais complexa. A matriz de peso espacial $W$, utilizada neste trabalho, está baseada na idéia dos $k$ vizinhos mais próximos. O cálculo utiliza a idéia de grande círculo entre os centros das regiões. A escolha da matriz de peso é muito importante em uma análise AEDE, pois todos os passos subseqüentes (ou resultados) dependerão dessa seleção. ${ }^{4}$

A forma da matriz de pesos espaciais é a seguinte:

$$
\left\{\begin{array}{l}
w_{i j}(k)=0 \text { se } i=j \\
w_{i j}(k)=1 \text { se } d_{i j} \leq D_{i}(k) \text { e } w_{i j}(k) / \sum_{j} w_{i j}(k) \text { para } k=1,2,(\ldots), n \\
w_{i j}(k)=0 \text { se } d_{i j}>D_{i}(k)
\end{array}\right.
$$

onde $d_{i j}$ é a distância, medida pelo grande círculo, entre os centros da regiões $i$ e $j$ e $D_{i}(k)$ é um valor crítico que define o valor de corte, ou seja, a distância máxima para considerar regiões vizinhas à região $i$. Isto significa que distâncias acima desse ponto não serão levadas em consideração, não sendo tomadas como vizinhas.

Segundo Le Gallo e Ertur (2003), a escolha de um número fixo de vizinhos mais próximos, em vez do uso de uma matriz simples de continuidade, é melhor, pois evita alguns problemas metodológicos possíveis em função da variação no número de vizinhos.

\section{BANCO DE DADOS E RESULTADOS EMPÍRICOS5}

\subsection{Banco de dados}

Cabe ressaltar que não há uma série temporal longa de matrizes de comércio inter-regional. Portanto, este trabalho utiliza os dados das Balanças Comerciais Interestaduais publicados pela Secretaria de Economia e Finanças do Ministério da Fazenda, com base em dados da Guia de Informação e Apuração do ICMS (GIA). Essa base de dados fornece informações sobre operações 
tributadas e operações globais (tributadas, isentas etc.) das entradas e saídas de mercadorias transacionadas bilateralmente pelas unidades da Federação. Para o ano de 1985, as informações da Balança Comercial das unidades da Federação foram obtidas junto ao IBGE, mais precisamente na Revista de Finanças Públicas. Já para o ano de 1997, as informações de exportações e importações interestaduais foram obtidas no site do Ministério da Fazenda, especificamente no site do Conselho Nacional de Política Fazendária (CONFAZ), na publicação da Balança Comercial Interestadual.

A utilização de tais variáveis deve ser realizada com uma certa cautela, pois existem algumas limitações, como, por exemplo, (a) o preenchimento correto das Guias de Informação e Apuração do ICMS, (b) o levantamento parcial das informações (cerca de 70\% do universo) e (c) a subestimação das operações com produtos agrícolas. Entretanto, mesmo com a existência de restrições na base de dados, é importante ressaltar que elas não se tornam um impeditivo para a implementação da análise da estrutura de comércio inter-regional das unidades da Federação como proposta neste trabalho. A possibilidade de melhor entender a dinâmica espacial das interações entre as unidades da Federação mais do que compensa as limitações da base de dados.

O tratamento implementado na base de dados foi o seguinte: (a) os dados de 1985 (Cr\$ bilhões) foram corrigidos para Reais (valores de 1997); (b) os dados de 1997 não sofreram modificações em termos de correção; e (c) os dados de 1985 e 1997 foram logaritmizados.

\subsection{Autocorrelação espacial global}

A tabela 1 mostra os resultados para a estatística $I$ de Moran para o comércio inter-regional e seus componentes (exportações e importações) nos anos de 1985 e 1997. Todos os coeficientes são positivos e estatisticamente significantes ( $p$-value 0,001 ), tomando por base 10 mil permutações aleatórias (Anselin, 1995). Os coeficientes de autocorrelação espacial global para o comércio inter-regional e seus componentes são significantes. Portanto, é possível afirmar que a distribuição forma clusters em ambos os períodos. Em outras palavras, as regiões com valores de comércio altos (baixos) estão localizadas próximo a outras regiões com valores de comércio igualmente altos (baixos). Esse padrão ocorre mais freqüentemente do que se as localizações fossem tomadas de forma aleatória. Para o ano de 1985, a 
Tabela 1: Estatística / de Moran para comércio e seus componentes (1985 e 1997)

\begin{tabular}{ccc}
\hline Ano & Variável & Moran's I \\
\hline 1985 & Comércio & 0,4772 \\
\hline & Exportação & 0,4679 \\
\hline 1997 & Importação & 0,4812 \\
\hline & & 0,5165 \\
\hline & Comércio & 0,4991 \\
\hline & Exportação & 0,5124 \\
\hline
\end{tabular}

Nota: Todas as estatísticas são significantes em $p=0,001$

autocorrelação espacial é significante, mas menos intensa. ${ }^{4}$ Isso se explica, num primeiro momento, pela intensificação do comércio durante o período, indicando um aumento da integração entre as regiões brasileiras. É importante afirmar que, mesmo com esta diferença na intensidade entre os coeficientes de autocorrelação espacial, a hipótese de aleatoriedade espacial é rejeitada para todas as variáveis nos dois períodos.

Cabe ressaltar que a estatística $I$ de Moran, empregando o conceito de vizinhos mais próximos para os valores de vizinhos utilizado neste trabalho (i.e., três, quatro e cinco), leva ao mesmo resultado em relação ao sinal e à significância da autocorrelação espacial, isto é, os resultados são robustos em relação à escolha da matriz de pesos. ${ }^{5}$

\subsection{Autocorrelação espacial local}

A análise de autocorrelação espacial local será realizada a partir do diagrama de dispersão de Moran e dos indicadores locais de associação espacial (LISA).

\subsubsection{Diagrama de dispersão de Moran}

As figuras 1 e 2 mostram os resultados dos diagrama de dispersão de Moran para o comércio inter-regional e seus componentes, para os anos de 1985 e 1997, com $k=5$ vizinhos mais próximos. Os resultados de autocorrelação espacial (Moran's I) apresentados na seção anterior podem ser corroborados pelo fato de que a maioria das regiões está localizada nos quadrantes $A A$ e BB. Para o comércio inter-regional como um todo, no ano de 1997, observase que $81,48 \%$ das unidades da Federação são caracterizadas pela presença de valores similares (37,04\% no quadrante $A A$ e $44,44 \%$ no quadrante $B B$ ). No que tange às regiões atípicas, isto é, regiões que apresentam desvios em relação ao padrão global de autocorrelação positiva, verifica-se que, para o 
Figura 1: Diagrama de Dispersão de Moran - comércio e seus componentes (1985)
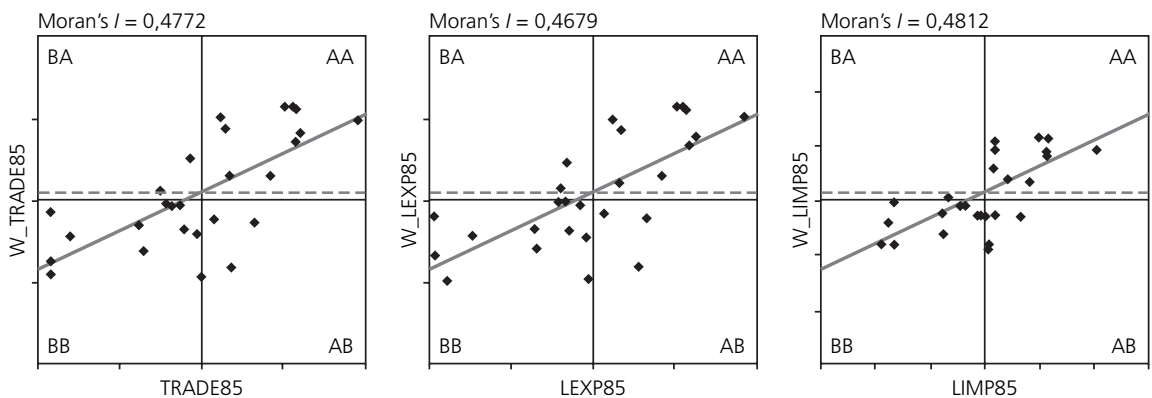

Fonte: Elaboração dos autores com a utilização do software GeoDA ${ }^{\mathrm{TM}}$.

Obs: trade85, lexp85 e limp85 - comércio, exportações e importações inter-regionais para o ano de 1985.

w_trade85, w_lexp85 e w_limp85- defasagem espacial do comércio, exportações e importaçōes inter-regionais para o ano de 1985.

Figura 2: Diagrama de dispersão de Moran - comércio e seus componentes (1997)
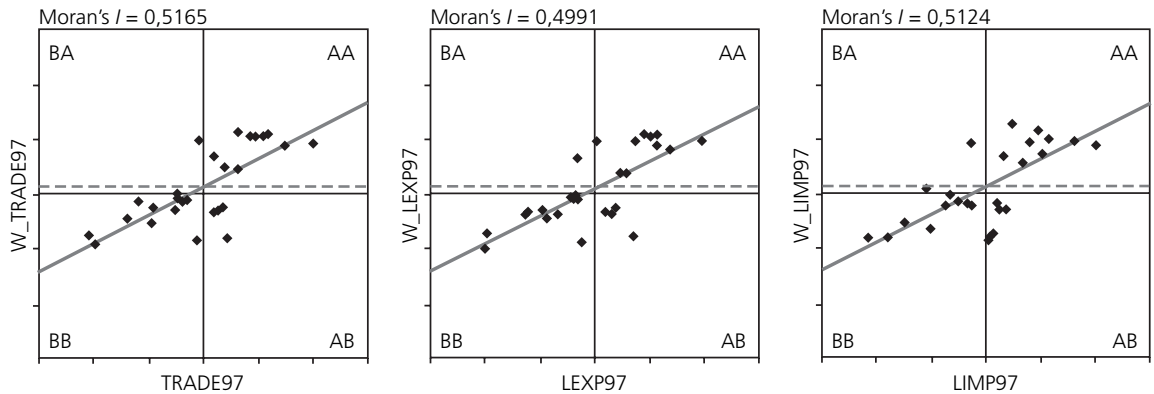

Fonte: Elaboração dos autores com a utilização do software GeoDATM.

Obs: trade97, lexp97 e limp97 - comércio, exportações e importações inter-regionais para o ano de 1997.

w_trade97, w_lexp97 e w_limp97- defasagem espacial do comércio, exportações e importações inter- regionais para o ano de 1997.

ano de 1997, cinco regiões tiveram associação com valores diferentes (uma no quadrante $B A$ e quatro no quadrante $A B)$.

Todos os resultados para o ano de 1997 apontam para a existência de heterogeneidade espacial na forma de três regimes espaciais diferentes. O primeiro corresponde ao quadrante $A A$. Ele é formado pelos estados brasileiros localizados nas regiões Sudeste e Sul (uma das áreas mais desenvolvidas do País), Bahia (um dos principais pólos de desenvolvimento da macrorregião Nordeste), Goiás (um dos estados que compõem a nova fronteira agrícola do País) e Distrito Federal. ${ }^{6} \mathrm{O}$ segundo regime corresponde ao quadrante $B B$ e inclui os estados localizados na região Norte (exceto o Amazonas) e os estados de Alagoas, Sergipe, Maranhão, Rio Grande do Norte, 
Piauí e Paraíba, na região Nordeste do País. Esse regime é formado pelos estados com menor tradição de comércio. O terceiro regime é formado pelos estados do Ceará, Pernambuco (região Nordeste), Amazonas (região Norte) e Mato Grosso (região Centro-Oeste), localizados no quadrante $A B$. Esses estados apresentam uma alta instabilidade espacial.

Para as exportações, no ano de 1997, também é possível apontar três regimes espaciais: (a) o primeiro, formado pelo quadrante $A A$, abrange os estados localizados no Sudeste, Sul e os estados de Goiás e Bahia; (b) o segundo, constituído por observações localizadas no quadrante $B B$, é formado pelos estados da região Norte (exceto Amazonas) e da região Nordeste (exceto Ceará, Pernambuco e Bahia); e (c) o terceiro regime está localizado no quadrante $A B$ e é composto pelos estados do Amazonas, Pernambuco, Ceará e Mato Grosso. Os resultados para as importações, em 1997, permitem afirmar que também existem três regimes (ver figura 2).

Uma comparação entre os resultados dos anos de 1985 e 1997 mostra que os clusters de comércio não sofreram grandes modificações. Desse modo, unidades da Federação pertencentes ao regime $B B$ em 1985 permaneceram nesse regime em 1997. Isso também ocorre com o regime $A A$, o que mostra uma certa estabilidade na estrutura de comércio inter-regional das unidades da Federação.

A inclinação positiva da curva apresentada no diagrama de dispersão de Moran confirma a existência de autocorrelação espacial. A inspeção do diagrama de dispersão de Moran (figuras 1 e 2) permite identificar tanto outliers quanto pontos de alavancagem.

De acordo com Varga (1998), localizações que são extremas à tendência central - ou seja, aquelas que não seguem o mesmo padrão de dependência espacial da maioria das observações - podem ser identificadas como outliers. Por outro lado, observações que têm grande influência sobre a tendência central são classificadas como pontos de alavancagem. Para definir os outliers e pontos de alavancagem, Varga (1998) faz uso da seguinte regra: se a reta de regressão de $W z$ contra $z$ tiver inclinação positiva, são consideradas outliers as observações que se situam a mais de dois desvios padrão do centro dos quadrantes e se localizam nos quadrantes $B A$ e $A B$. Por outro lado, aquelas observações que estão a mais de dois desvios padrão do centro dos quadrantes e se localizam nos quadrantes $A A$ e $B B$ são pontos de alavancagem. 
As figuras 3 e 4 mostram a influência dos pontos de alavancagem sobre a tendência central. Nestas figuras o Estado de São Paulo foi considerado ponto de alavancagem ${ }^{7}$ e foi retirado da amostra para verificar qual o padrão de comércio inter-regional sem a participação de São Paulo. Percebe-se que tanto para o ano de 1985 quanto para o ano de 1997 há um aumento no I de Moran, à exceção do resultado para as exportações de 1985. Esse resultado pode ser interpretado da seguinte maneira: ao se retirar o Estado de São Paulo, o índice I de Moran aumenta, mostrando que há maior integração entre as unidades da Federação. Em outras palavras, se não houves-

Figura 3: Diagrama de dispersão de Moran - comparação do I de Moran (1985)
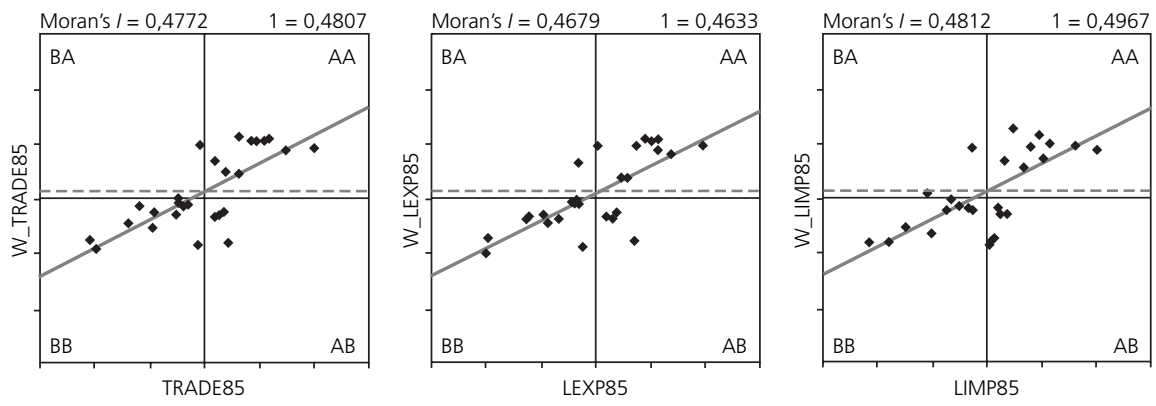

Fonte: Elaboração dos autores com a utilização do software GeoDA ${ }^{\mathrm{TM}}$.

Obs: trade85, lexp85 e limp85 - comércio, exportações e importaçōes inter-regionais para o ano de 1985.

w_trade85, w_lexp85 e w_limp85- defasagem espacial do comércio, exportações e importações inter-regionais para o ano de 1985.

Figura 4: Diagrama de dispersão de Moran - comparação do / de Moran (1997)
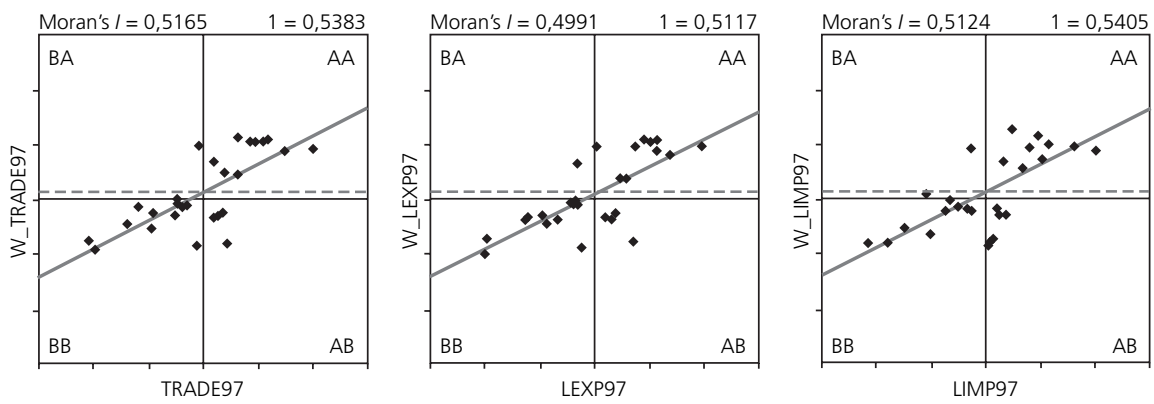

Fonte: Elaboração dos autores com a utilização do software GeoDATM.

Obs: trade97, lexp97 e limp97 - comércio, exportações e importações inter-regionais para o ano de 1997.

W_trade97, w_lexp97 e w_limp97-defasagem espacial do comércio, exportações e importações inter-regionais para o ano de 1997. 
se, de forma hipotética, comércio com o Estado de São Paulo, haveria um maior efeito vizinhança no comércio inter-regional e seus componentes. Dessa forma, isso pode indicar o grau de polarização exercido pela economia paulista.

\subsubsection{Mapas de dispersão de Moran}

Os resultados descritos anteriormente para o ano de 1997 podem ser visualizados nas figuras 5 a 7, nos Mapas de Dispersão de Moran, os quais possibilitam uma visão mais clara da dependência espacial. Para o comércio como um todo, pode-se observar que o cluster formado pelos valores altoalto está localizado na porção centro-sul do País. Essa parte do País apresenta a melhor rede de transporte, um ótimo número de cidades médias e também a mais completa rede de universidades e centros de pesquisa. Em outras palavras, a região pode ser classificada como a parte do País que tem as melhores condições de se desenvolver no curto prazo. Nessa região estão as áreas metropolitanas do Rio de Janeiro, São Paulo, Belo Horizonte, Campinas, Curitiba e Porto Alegre que, como afirmado por Diniz (2002), representam as principais alternativas para a expansão industrial e produtiva do Brasil. O regime baixo-baixo está localizado, principalmente, no Norte do País e apresenta algumas descontinuidades na região Nordeste.

A figura 8 mostra o Mapa de Dispersão de Moran para o comércio inter-regional no ano de 1985. É possível observar que durante o período de análise houve um decréscimo no número de estados localizados no regime $A A$, ou seja, esse regime tornou-se mais concentrado na parte centro-sul do País. O crescimento do regime $B B$ (valores abaixo da média do comércio inter-regional) no Norte e no Nordeste do país pode corroborar tal resultado. Pode-se afirmar que o aumento na complexidade do setor produtivo e o papel relevante desempenhado pelas economias de escala, como determinantes do comércio, levam à concentração do comércio em determinadas localidades (Helpman e Krugman, 1985; Krugman, 1991). No caso brasileiro, durante o período de análise, o fenômeno está ocorrendo nas regiões Sudeste e Sul do país, responsáveis por mais de 70\% do PIB nacional. Dado que o comércio é uma função da renda e do produto, mesmo com uma relativa desconcentração da produção industrial nas cidades do Rio de Janeiro e São Paulo, os resultados encontrados mostram que há uma tendência de 
Figura 5: Brasil - Mapa de Dispersão de Moran para o comércio inter-regional, 1997

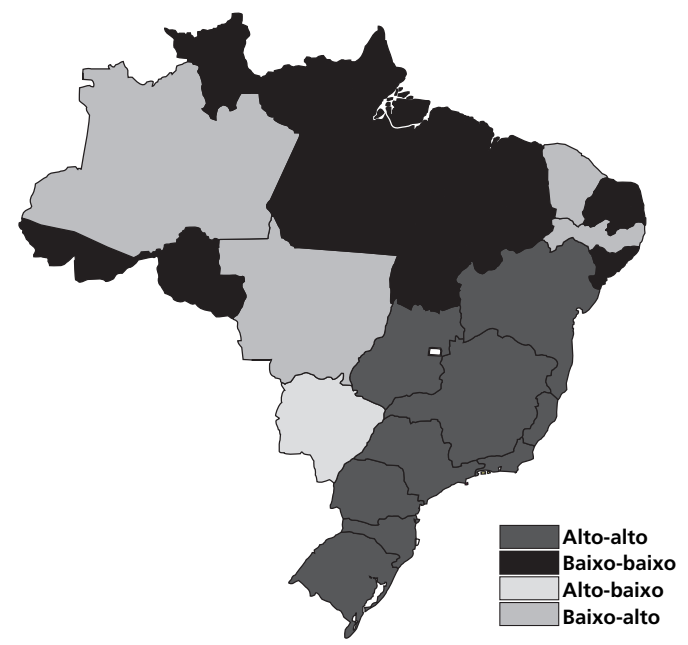

Fonte: Elaboração dos autores com a utilização do software GeoDA ${ }^{\mathrm{TM}}$

Figura 6: Brasil - Mapa de Dispersão de Moran para as exportações inter-regionais, 1997

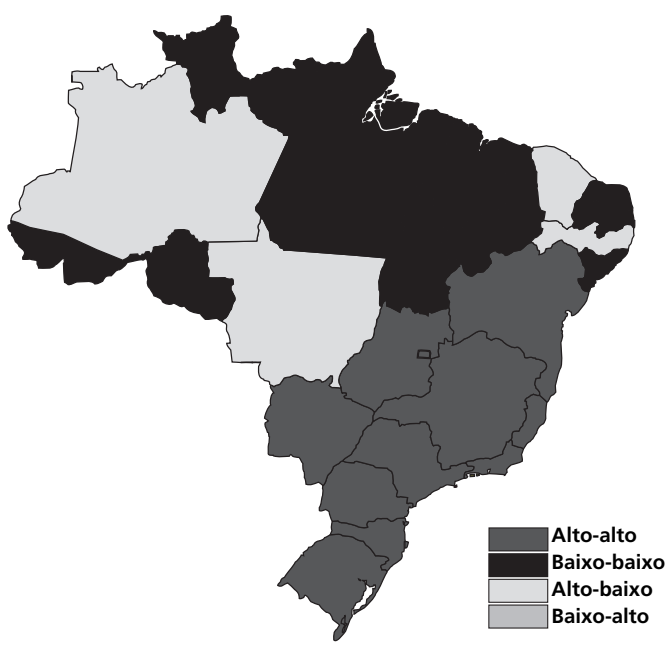

Fonte: Elaboração dos autores com a utilização do software GeoDA ${ }^{\mathrm{TM}}$ 
Figura 7: Brasil - Mapa de Dispersão de Moran para importações inter-regionais, 1997

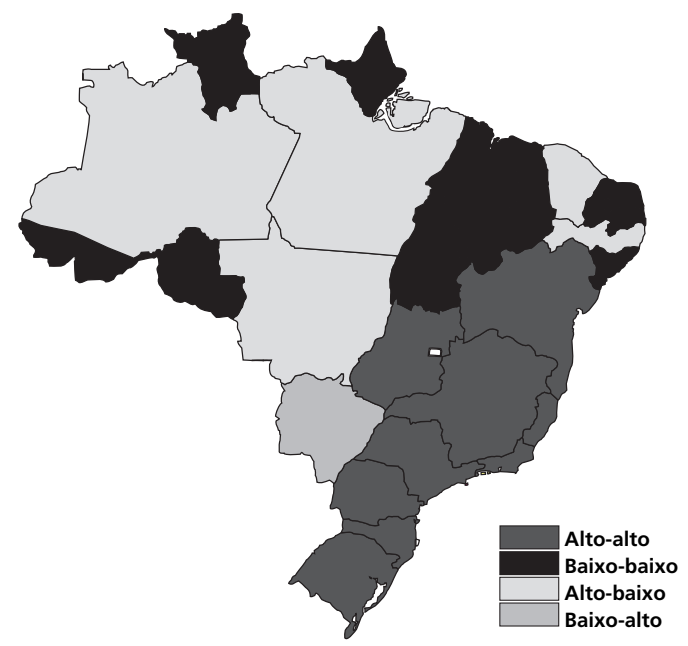

Fonte: Elaboração dos autores com a utilização do software GeoDA ${ }^{\mathrm{TM}}$

Figura 8: Brasil - Mapa de Dispersão de Moran para o comércio inter-regional, 1985

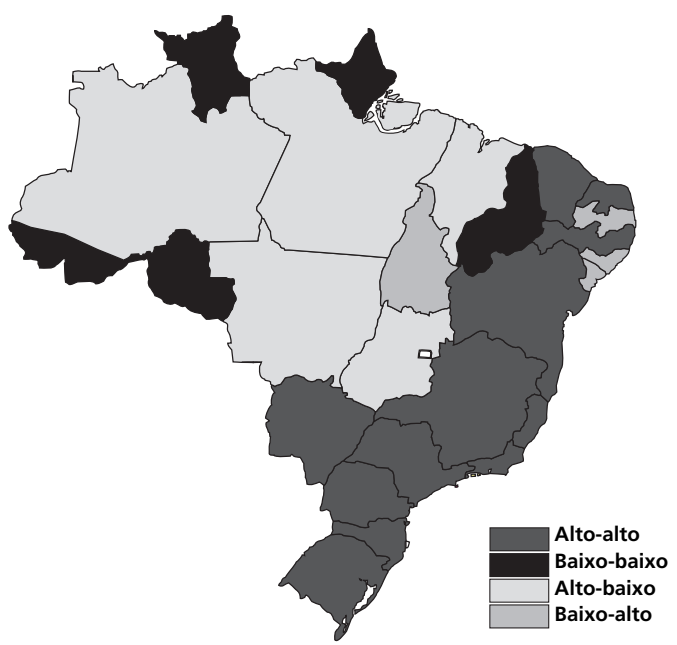


reconcentração espacial, em termos de comércio, na macrorregião Sudeste e Sul do país. Essa tendência pode estar baseada, em parte, no desenvolvimento das metrópoles de segundo nível e das cidades médias. Elas representam as áreas com grande probabilidade de crescimento econômico e industrial, levando a um aumento da renda e do produto total e, como conseqüência, a um aumento no comércio (interações).

Segundo dados apresentados por Diniz (2002), houve uma relocalização de algumas indústrias (têxtil e alimentos) em direção ao Nordeste (em função de incentivos fiscais). Entretanto, a base da indústria nacional (metalurgia, mecânica, equipamentos eletrônicos e elétricos e química) ainda está concentrada no Sudeste-Sul do País. Aliando a discussão acima aos resultados encontrados, é possível afirmar que as interações entre os setores constituintes da base industrial nacional podem contribuir, em parte, para o aumento do comércio dentro da macrorregião Sudeste-Sul.

Conforme a literatura de comércio, pode-se utilizar a relação entre comércio e renda para melhor entender o movimento de concentração do regime $A A$. De acordo com o IBGE (2004), a diferença entre a maior renda per capita e a mais baixa, para o ano de 2001, foi de $88 \%$ e ocorreu entre as regiões Centro-Oeste (Distrito Federal) e Nordeste do País. Já a distribuição espacial do PIB, para 2001, mostra que (a) os estados localizados no Sul-Sudeste mais o Distrito Federal, Mato Grosso do Sul e Amazonas são responsáveis pelos maiores níveis de PIB per capita (> R \$6.000,00 por ano); (b) o Nordeste (Sergipe), o Norte (Rondônia e Amapá) e o Centro-Oeste (Goiás e Mato Grosso) apresentam um PIB per capita entre R \$ 4.000,00 e R\$ 6.000,00; e (c) Tocantins, Acre, Roraima e Pará e os estados do Nordeste (à exceção de Sergipe) apresentam o menor nível de PIB per capita $(<\mathrm{R} \$ 4.000,00$ por ano).

\subsubsection{Indicadores locais de associação espacial (LISA)}

Os indicadores locais de associação espacial (LISA) para o comércio inter-regional brasileiro e seus componentes estão representados nos Mapas de Significância de Moran — mapas de clusters (figuras 9 a 12) —, utilizando o nível de pseudo-significância de $5 \%$ de Bonferroni. ${ }^{8}$ Ao se examinarem os mapas, percebe-se que existem alguns clusters locais que merecem maior atenção. 
Primeiramente, é possível sustentar que o padrão local de associação espacial reflete o padrão global, no que se refere à autocorrelação espacial positiva. Ao se utilizar o pseudonível de significância de 5\% de Bonferroni, $84,6 \%$ dos LISAs significantes para o comércio inter-regional como um todo situam-se nos quadrantes $A A$ e $B B$ do gráfico de dispersão. Pode-se também enfatizar que há um desequilíbrio na distribuição entre os quadrantes $A A$ e $B B$, pois $63,6 \%$ das regiões estão localizadas no quadrante $A A$. Portanto, conclui-se que há, de forma preponderante, regiões com alto comércio vizinhas a regiões com alto nível de comércio. Tais regiões estão localizadas no centro-sul do País (de Minas Gerais até o Rio Grande do Sul).

A persistência desse padrão pode ser explicada, dentre outros fatores, pelos spillovers de São Paulo para os estados vizinhos. São Paulo tem um importante papel como pólo de atração. A área metropolitana de São Paulo começa a apresentar deseconomias de escala no fim dos anos 1980 e, em conseqüência, há um crescimento das áreas vizinhas. Assim, tal fenômeno pode ter contribuído para o aumento do comércio na região (figura 9).

Um segundo ponto é que os desvios em relação à tendência global são realmente limitados. Eles representam apenas $15,4 \%$ dos LISAs significantes. Para o comércio inter-regional, observa-se a existência de uma região $A B$, representada pelo Estado do Amazonas. É importante salientar a heterogeneidade da estrutura produtiva do Estado do Amazonas em relação aos demais estados da macrorregião, uma vez que ela é formada principalmente pela indústria de componentes eletrônicos. Isso pode, em grande parcela, dificultar o aumento da interação dentro da macrorregião Norte. Por outro lado, o Estado do Mato Grosso do Sul é classificado com BA (figura 9).

$\mathrm{Na}$ figura 10 é possível observar os resultados da estatística LISA, para as exportações, para o ano de 1997. As principais características consistem em: (a) padrão local de associação espacial que reflete a tendência global em direção à autocorrelação espacial positiva, visto que 91,6\% do LISA significante encontra-se nos quadrantes $A A$ e $B B$ do gráfico de dispersão; (b) desigualdade na distribuição entre os regimes $A A$ e $B B$, já que a maioria das regiões está localizada no quadrante $A A$; (c) regiões formadas por estados com alta exportação vizinhas de estados com altos valores para exportação e concentradas na parte centro-sul do País; e (d) estados que apresentam exportações abaixo da média, localizados no norte do Brasil. Portanto, para as 
Figura 9: Mapa de cluster para o comércio inter-regional brasileiro, $1997^{11}$
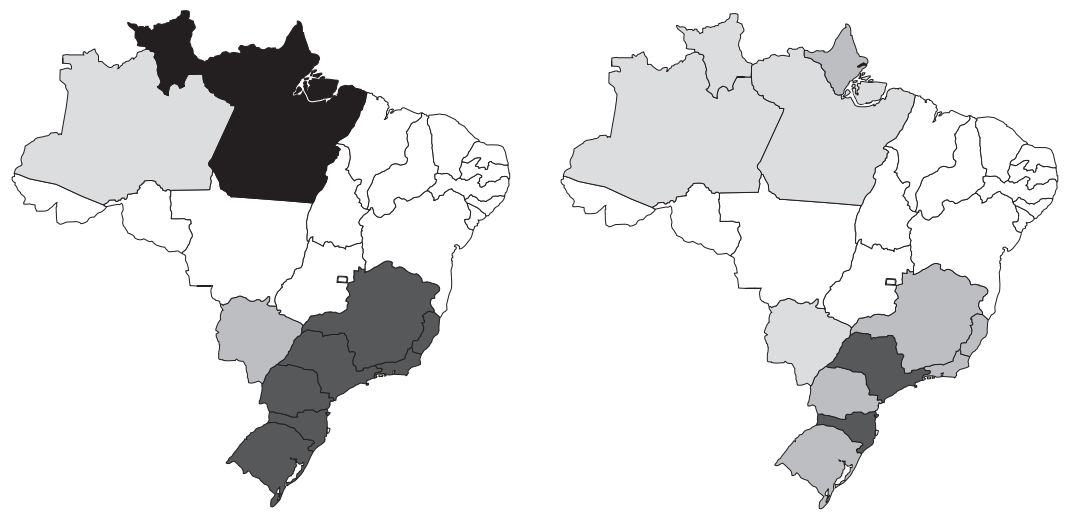

Fonte: Elaboração própria dos autores.

Figura 10: Mapa de cluster para as exportações inter-regionais brasileiras, $1997^{11}$
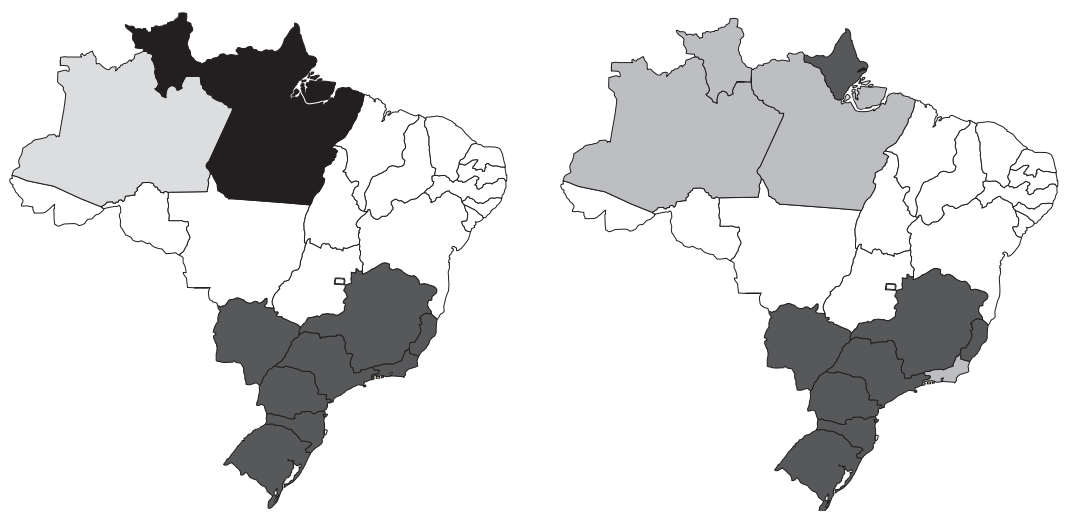

Fonte: Elaboração própria dos autores.

exportações, torna-se possível classificar o padrão apresentado como sendo de centro (centro-sul) e periferia (norte).

Os resultados para a importação inter-regional podem ser examinados na figura 11. A estrutura de distribuição de estados com $A A$ no centro-sul e estados com $B B$ no norte do País também ocorre para as importações interregionais. Um resultado muito interessante é observado para o Distrito Federal. Ele é classificado como $A A$ e o resultado pode ser explicado por meio da análise da estrutura produtiva do Distrito Federal, o qual tem o setor de 
Figura 11: Mapa de cluster para as importações inter-regionais brasileiras, $1997^{11}$
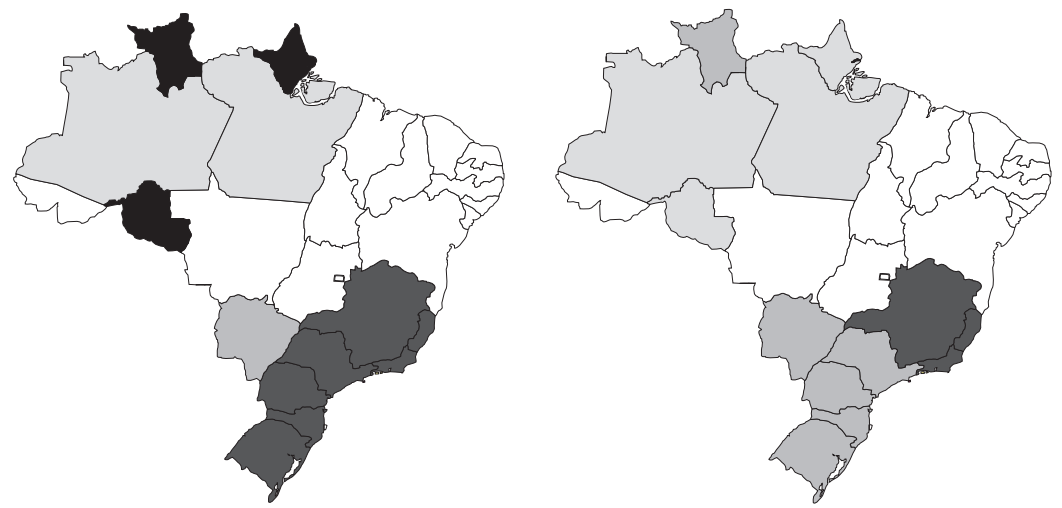

Fonte: Elaboração própria dos autores.

Figura 12: Mapa de cluster para o comércio inter-regional brasileiro, $1985^{11}$
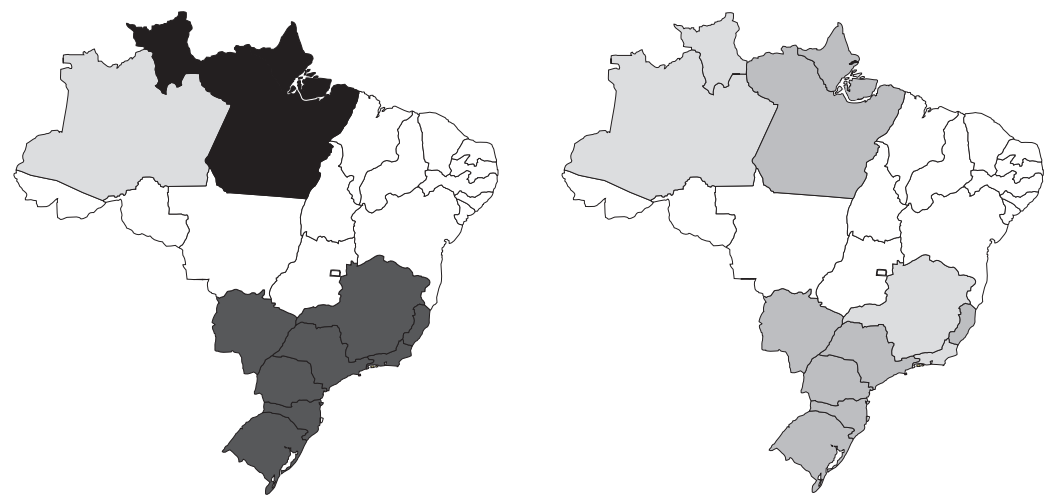

Fonte: Elaboração própria dos autores.

serviços como a principal atividade e apresenta um pequeno setor industrial e agrícola (i.e., pequena participação na formação do produto regional).

Comparando as figuras 9 e 12, é possível verificar se os clusters de comércio regional persistem ao longo do tempo. O cluster de $A A$ torna-se menor e, em 1997, é formado exclusivamente por estados localizados nas regiões Sudeste e Sul. Já o cluster BB é constituído por estados pertencentes à região Norte. Logo, isso pode indicar um aumento das disparidades entre o Norte e Sul do Brasil. 


\subsubsection{Diagrama de Dispersão de Moran Bivariado}

O Diagrama de Dispersão de Moran Bivariado tem por objetivo analisar a autocorrelação espacial num determinado espaço de tempo. Assim, nesta subseção buscar-se-á comprovar as hipóteses, apresentadas nas subseções anteriores, de que a estrutura de comércio entre as unidades da Federação não se modifica ao longo do período analisado. O diagrama de dispersão é formado por duas variáveis iguais, neste caso, comércio inter-regional, exportações e importações, mas mensuradas em diferentes períodos (figura 13). Os resultados do Diagrama de Dispersão de Moran Bivariado podem ser mais bem visualizados nas figuras 14, 15 e 16 .

Ao analisar a figura 13, verifica-se que existe uma autocorrelação espacial positiva temporal para o comércio inter-regional e para seus componentes. Isso significa que unidades da Federação com um alto índice de comércio inter-regional em 1985 continuam com o mesmo padrão em 1997 e, do mesmo modo, unidades da Federação que tinham um baixo índice de comércio inter-regional em 1985 permanecem com o mesmo padrão em 1997. Em outras palavras, não houve mudanças na estrutura espacial do comércio inter-regional e de seus componentes para o período de análise.

As figuras 14, 15 e 16 mostram os mapas de clusters bivariados. Percebese que tanto para comércio inter-regional quanto para exportações e importações, há um padrão dicotômico, ou seja, o cluster formado por regiões alto-alto situa-se no centro-sul do País e o cluster formado por regiões bai-

Figura 13: Diagrama de Dispersão de Moran Bivariado - comércio, exportações e importações
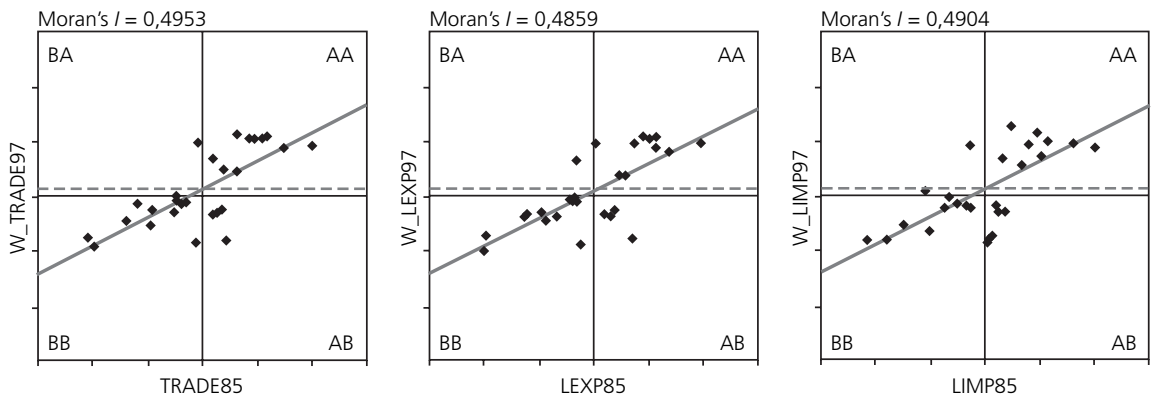

Fonte: Elaboração dos autores com a utilização do software GeoDA ${ }^{\mathrm{TM}}$.

Obs: trade85, lexp85 e limp85 - comércio, exportações e importações inter-regionais para o ano de 1985.

w_trade97, w_lexp97 e w_limp97-defasagem espacial do comércio, exportações e importações inter- regionais para o ano de 1997. 
xo-baixo está localizado na região Norte do País. Os resultados intertemporais corroboram a hipótese de manutenção da estrutura concentracionista das interações entre as unidades da Federação. Assim sendo, é possível afirmar, com base nos resultados do presente artigo, que a dinâmica espacial do comércio interestadual brasileiro não sofreu grandes modificações ao longo do período em questão. Portanto, o efeito vizinhança positivo para comércio, apontado por Domingues et al. (2002), se verifica em maior grau na macrorregião Sudeste-Sul, ou seja, na região de localização do cluster AA.

Figura 14: Mapas de Clusters Bivariados - comércio inter-regional, em 1997 e $1985^{12}$
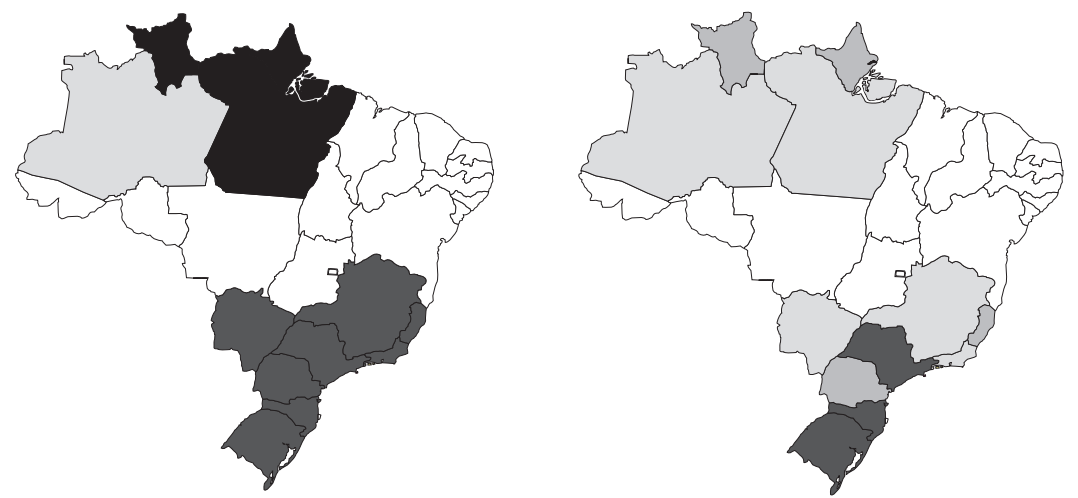

Fonte: Elaboração própria dos autores.

Figura 15: Mapas de Clusters Bivariados - Exportações em 1997 e $1985^{12}$
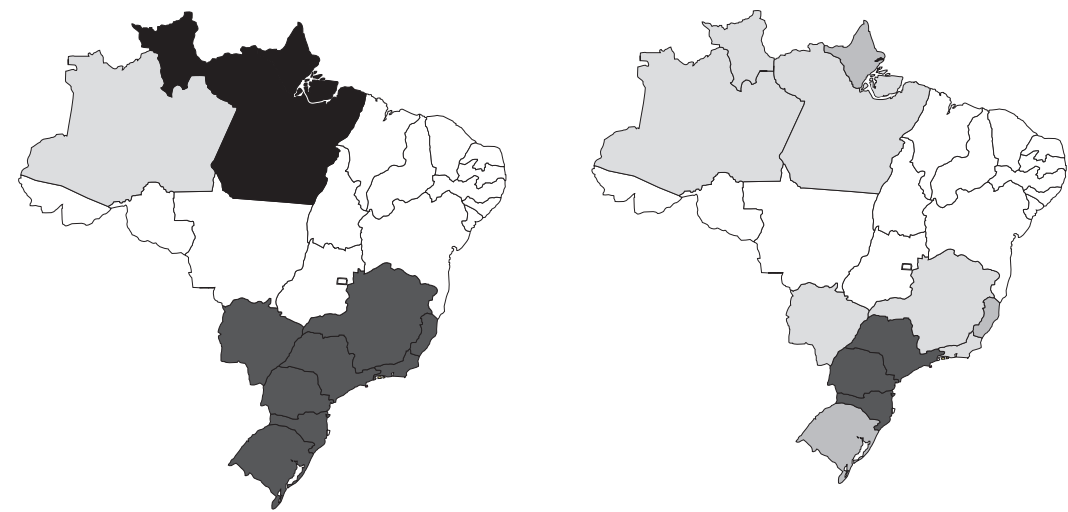
Figura 16: Mapas de Clusters Bivariados - Importações em 1997 e $1985^{12}$
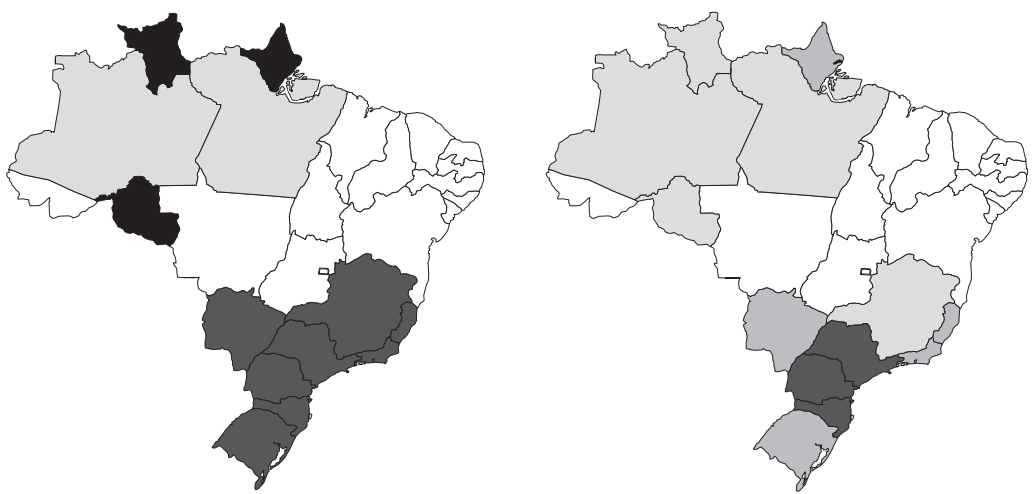

Fonte: Elaboração própria dos autores.

\subsection{Análise da robustez utilizando a matriz de transição de probabilidades}

Considera-se robusto o resultado que não se modifica quando o número de vizinhos se altera. Segundo Le Gallo e Ertur (2003: 15), "os resultados são robustos se uma região que se situa em um estado particular quando a análise é implementada para a matriz de $k$ vizinhos (i.e., não significante - NS, AA, BB, AB, BA) permanece no mesmo estado para outras matrizes de pesos espaciais". ${ }^{9}$ Visando a tal objetivo, os autores sugerem o uso da estrutura das matrizes de transição, pois elas medem a transição entre diferentes distribuições em um dado período. ${ }^{10}$

A tabela 2 mostra duas matrizes de transição de probabilidades diferentes. Elas são utilizadas para verificar a robustez das estatísticas LISA para o comércio, no ano de 1997, utilizando o nível de significância de 5\% de Bonferroni. ${ }^{11}$ Tais resultados mostram que a região associada com um LISA significante permanece no mesmo estado, não importando o número de vizinhos escolhidos. Portanto, pode-se afirmar que os resultados são robustos não importando a matriz de peso escolhida.

\section{CONSIDERAÇÕES FINAIS}

A motivação deste trabalho foi entender a estrutura de comércio entre os estados brasileiros. A aplicação da análise exploratória dos dados espaciais 
Tabela 2: Análise de robustez para a estatística LISA (comércio inter-regional - 1997)

\begin{tabular}{ccccccc}
\hline & K $=4$ & NS & AA & BB & BA & AB \\
\hline & NS & $\mathbf{1 0 0 . 0 0}$ & 0.00 & 0.00 & 0.00 & 0.00 \\
\hline K=5 & AA & 14.29 & $\mathbf{8 5 . 7 1}$ & 0.00 & 0.00 & 0.00 \\
\hline & BB & 66.67 & 0.00 & $\mathbf{3 3 . 3 3}$ & 0.00 & 0.00 \\
\hline & BA & 0.00 & 0.00 & 0.00 & $\mathbf{1 0 0 . 0 0}$ & 0.00 \\
\hline & AB & 0.00 & 0.00 & 0.00 & 0.00 & $\mathbf{1 0 0 . 0 0}$ \\
\hline & & & & & BA & AB \\
\hline & K $=3$ & NS & AA & BB & 0.00 & 0.00 \\
\hline KS $=5$ & $\mathbf{1 0 0 . 0 0}$ & 0.00 & 0.00 & 0.00 & 0.00 \\
\hline & AA & 0.00 & $\mathbf{1 0 0 . 0 0}$ & 0.00 & 0.00 & 0.00 \\
\hline & BB & 33.33 & 0.00 & $\mathbf{6 6 . 6 7}$ & $\mathbf{1 0 0 . 0 0}$ & 0.00 \\
\hline & BA & 0.00 & 0.00 & 0.00 & 0.00 & $\mathbf{1 0 0 . 0 0}$ \\
\hline
\end{tabular}

nos fluxos de comércio permite afirmar que há autocorrelação espacial positiva, a qual persiste durante o período de análise, ou seja, as regiões com comércio alto (baixo) estão e permanecem localizadas junto às regiões com comércio alto (baixo). A análise do valor padronizado da estatística I de Moran demonstra que a estrutura de comércio interestadual brasileira torna-se mais intensa durante o período de estudo.

Os resultados para o LISA confirmam a presença significante e a persistência ao longo do tempo da autocorrelação espacial na forma de dois clusters espaciais distintos, de valores altos e baixos para o comércio e seus componentes.

Em síntese, a partir da análise exploratória dos dados espaciais pode-se apontar que: (a) há um aumento do comércio interestadual no período; (b) existe uma heterogeneidade espacial no comércio interestadual, sendo que o cluster formado por valores de comércio alto localiza-se na porção centrosul do País e o cluster formado por unidades da Federação com comércio abaixo da média vizinhas de unidades da Federação com o mesmo padrão localiza-se na região Norte; e (c) a análise intertemporal mostra que tal padrão não se modifica ao longo do período analisado. O prosseguimento de tal padrão nos próximos períodos torna possível inferir sobre o aumento das disparidades regionais no Brasil.

Portanto, pergunta-se: qual a alternativa para minimizar esse quadro? Antes de discutir tal indagação, cabe ressaltar a importância dos resultados encontrados no presente artigo. A definição de políticas públicas objetivan- 
do o desenvolvimento regional depende do maior conhecimento das especificidades das regiões e das delimitações precisas do espaço a ser abordado. Logo, a apresentação de informações sobre a dinâmica espacial do comércio neste trabalho - isto é, a análise detalhada das especificidades das unidades da Federação no que se refere à capacidade e ao grau (e.g. alto-alto, baixobaixo, alto-baixo e baixo-alto) de interação das mesmas - possibilita uma tomada de decisões mais precisa por parte dos agentes públicos.

Neste sentido, políticas que produzam uma desconcentração produtiva e, em conseqüência, descentralizem a estrutura de comércio no Brasil são bem-vindas. Essas políticas passam por investimentos públicos em infra-estrutura econômica e social, oferta de linhas de crédito às iniciativas privadas de investimento e fortalecimento do desenvolvimento local como forma de promover a ocupação econômica do território nacional. Em outras palavras, programas de dinamização e/ou reorganização da estrutura de transportes (i.e., estudos para a implementação mais efetiva de transporte multimodal, recuperação das estradas, revitalização de portos na região Nordeste, dentre outros) e o fortalecimento dos arranjos produtivos locais e/ou clusters — tais como o de vestuário na região Nordeste, calçados na região Sul, frutas na região Nordeste, turismo nas regiões Nordeste e Sul do País, dentre outros - podem gerar uma desconcentração produtiva e, portanto, reestruturar a dinâmica espacial do comércio inter-regional brasileiro.

\section{NOTAS}

1. Tobler (1979): "Everything is related to everything else, but near things are more related than distant things."

2. Os Lags espaciais ou defasagem espacial são obtidos pela multiplicação do vetor da variável de interesse $\left(z_{t}\right)$ pela matriz de pesos espaciais $(W)$. Desse modo, a variável de interesse é ponderada pelo valor dos vizinhos através da matriz de pesos espaciais.

3. "This means that when the overall significance associated with the multiple comparisons (correlated tests) is set to $a$, and there are $m$ comparisons, then the individual significance $a$ should be set to either $a / m$ (Bonferroni) or $1-(1-\alpha)^{1 / m}$ (Sidák)" (Anselin, 1995: 96).

4. Neste estudo foram também utilizados valores de $K=3$ e 4 a fim de verificar a robustez dos resultados (ver subseção 2.4).

5. Para implementação da metodologia, ver Anselin (1999). O software utilizado para implementar a metodologia foi o Spacestat. Esta seção segue, em parte, a apresentação feita por Haddad (2002). 
6. É importante verificar se essa integração está concentrada no território nacional. Essa questão pode ser analisada com mais detalhes quando for introduzido o resultado para autocorrelação espacial local, ou seja, ao verificar a existência de clusters de comércio ou regimes espaciais.

7. Todos os resultados estão disponíveis para consulta.

8. O volume de importações e as características de Brasília podem explicar o resultado. Segundo Diniz (2002), Brasília congrega as principais atividades da Administração Pública Federal, apresenta a maior renda per capita e é a região na qual o consumo desempenha um papel mais importante do que a produção.

9. Domingues et al. (2002) também encontraram um comportamento atípico para o Estado de São Paulo.

10. Para mais detalhes, ver Anselin (1995).

11. Obs. ${ }_{1}$ : A cor cinza-escuro representa os clusters alto-alto, a cor preta representa os clusters baixo-baixo, a cor cinza-médio identifica os clusters baixo-alto e, por fim, a cor cinza-claro identifica os clusters alto-baixo.

Obs. ${ }_{2}$ : No mapa de significância, a gradação do cinza-escuro até chegar ao cinza mais claro representa níveis de significância de $0,01 \%, 0,1 \%, 1 \%$ e 5\%, respectivamente.

12. Obs. ${ }_{1}$ : A cor cinza-escuro representa os clusters alto-alto, a preta os clusters baixo-baixo, a cinza-médio identifica os clusters baixo-alto e, por fim, a cor cinza-claro exibe os clusters alto-baixo.

Obs. 2 : No mapa de significância, a gradação do cinza-escuro até chegar ao cinza mais claro representa níveis de significância de $0,01 \%, 0,1 \%, 1 \%$ e 5\%, respectivamente.

13. "The results are robust if a region that is in a particular state with the $k$ neighbor matrix (i.e., not significant NS, HH, LL, HL, LH statistics) remains in this state for the other weight matrices." Le Gallo e Ertur (2003: 15).

14. Para mais aplicações da metodologia, ver Le Gallo (2003), Rey (2001) e Dall'erba (2003).

15. A análise de robustez da estatística LISA para exportações e importações para 1996 e 1985 está à disposição para consulta.

\section{REFERÊNCIAS BIBLIOGRÁFICAS}

ANSELIN, L. (1999) SpaceStat, a software package for the analysis of spatial data version 1.90. AnnArber: BioMedware.

(1998) "Interactive techniques and exploratory spatial data analysis". In: P. A. Longley, M. F. Goodchild, D. J. Maguire e D. W. Wind (eds.), Geographical Information Systems: principles, techniques, management and applications. Wiley: Nova York, p. 253-265.

(1996) "The Moran scatterplot as an ESDA tool to assess local instability in spatial association”. In: M. Fisher, H. J. Scholten, e D. Unwin (eds.), Spatial Analytical Perspectives in GIS. Londres: Taylor\&Francis, p. 111-125.

(1995) "Local indicators of spatial association - LISA". Geographical Analysis, v. 27 (2), April, p. 93-115. 
CONFAZ. Balança Comercial Interestadual. Disponível em www.fazenda.gov.br

CLIFF, A. D., ORD, J. K. (1981) Spatial Processes: models and applications: Londres: Pion.

DALL'ERBA, S. (2003) "Distribution of regional income and regional funds in Europe 19891999: an exploratory spatial data analysis”. Discussion Paper REAL 03-T-1. UIUC.

DINIZ, C. C. (2002) “A nova configuração urbano-industrial no Brasil”. In: A. Kon (org.), Unidade e fragmentação: a questão regional no Brasil. São Paulo: Perspectiva.

—_ LEMOS, M. B. (1989) "Dinâmica regional e suas perspectivas no Brasil”. In: Para a década de 90: prioridades e perspectivas de políticas públicas. Brasília: IPEA -IPLAN, v. 3, p. 161-199.

DOMINGUES, E. P., HADDAD, E. H., HEWINGS, G. J. D., PEROBELLI, F. S. (2002) “Structural changes in the Brazilian interregional economic system, 1985-1997: holistic matrix interpretation". Australasian Journal of Regional Studies, v. 8, n. 1, p. 21-44.

ERTUR, C., LE GALLO, J. (2003) "An exploratory spatial data analysis of European regional disparities, 1980-1995”. In: Bernard Fingleton (ed.), European Regional Growth: advances in spatial science. Springer-Verlag, p. 55-98.

GALVÃO, O. A. (1993) “Comércio interestadual por vias internas e integração regional no Brasil”. Anais do XXI Encontro Nacional de Economia. Belo Horizonte: ANPEC, v. 1, p. 257-279.

HADDAD, E. A., AZZONI, C. R., DOMINGUES, E. P., PEROBELLI, F. S. (2002) “Macroeconomia dos estados e matriz interestadual de insumo-produto". Revista Economia Aplicada, 6 (4), out./dez.

HADDAD, M. A. (2002) "An exploratory spatial data analysis of human development in Brazil" (mimeo).

HELPMAN, E., KRUGMAN, P. R. (1985) Market Structure and Foreign Trade: increasing returns, imperfect competition, and the international economy. Cambridge, MA: MIT Press.

IBGE. Revista de Finanças Públicas, v. 47, n. 369.

KRUGMAN, P. R. (1991) Geography and Trade. Cambridge, MA: MIT Press.

LE GALLO, J., ERTUR, C. (2003) "Exploratory spatial data analysis of the distribution of regional per capita GDP in Europe, 1980-1995”. Papers in Regional Science, v. 82 (2) p. $175-201$.

LE GALLO, J. (2003) "Space-time analysis of GDP disparities among European regions: a Markov chains approach". International Regional Science Review, forfhcoming.

PACHECO, C. A. (1998) Fragmentação da nação. Campinas: Unicamp/IE.

REY, S. (2001) "Spatial empirics for economic growth e convergence". Geographical Analysis, v. 33 (3), July, p. 195-214.

TOBLER, W. (1979) “Cellular geography”. In: S. Gale e G. Olsson (eds.), Philosophy in Geography. Dordrecht: Reidel, p. 379-386.

VARGA, A. (1998) University Research and Regional Innovation: a spatial econometric analysis of academic technology transfers. Boston/Dordrecht/Londres: Kluwer Academic Publishers. 